\title{
Different SAFETY CERTIFIABLE CONCEPTS For MOBILE ROBOTS IN INDUSTRIAL ENVIRONMENTS
}

\author{
Maximilian Papa, David Kaselautzke, Kemajl Stuja \& Walter Wölfel
}
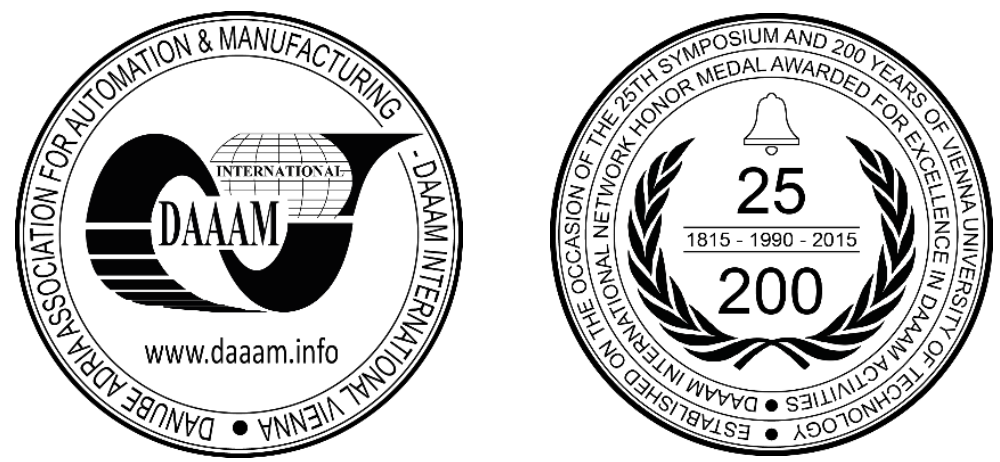

This Publication has to be referred as: Papa, M[aximilian]; Kaselautzke, D[avid]; Stuja, K[emajl] \& Wolfel, W[alter] (2018). Different Safety Certifiable Concepts for Mobile Robots in Industrial Environments, Proceedings of the 29th DAAAM International Symposium, pp.0791-0800, B. Katalinic (Ed.), Published by DAAAM International, ISBN 9783-902734-20-4, ISSN 1726-9679, Vienna, Austria

DOI: $10.2507 / 29$ th.daaam.proceedings.115

\begin{abstract}
Recent progress in mobile robotics paves the way of their usage in industrial environments. Nevertheless, they are currently no standards, which provide safety regulation in these environments for autonomous mobile robots. In this paper, three different safety concepts for mobile robots will be discussed, whereby the safe usage of a mobile robot in an industrial setting should be ensured. The first concept contains a safety ring, which allows the mobile robot to use probabilistic robotics in combination with a robust obstacle avoidance and safe hazard detection. Further, the second concept treats safe navigation without probabilistic robotics. The priority hereby lies on the self-localisation with reliable sensor methods. And in the third concept, an aware environment with object detection and tracking is shown. Virtual sensors in form of wireless sensor networks placed in the factory replace the need of physical sensors on mobile robots. The outline of this work is a theoretical comparison of these three safety concepts in consideration of their specific properties and their recommended usage for different scenarios.
\end{abstract}

Keywords: mobile robot; safety ring; safe navigation; aware environment; safe sensors

\section{Introduction}

Until now, simple automated guided vehicles (AGV) are getting used in industrial environments to fulfil simple transportation tasks. With the ongoing progress of new technologies in this area and especially in probabilistic robotics, the usage these approaches for more flexible mobile robots are possible. Autonomous mobile robots (AMR) getting more usage in industrial environments for logistical and manipulating tasks. Until now, a standard for mobile robots does not exist. Therefore, in cooperation with the department "MA23" of the city of Vienna and the "TÜV Austria" different safety concepts for mobile robot are in development.

Nearly all state-of-the-art mobile robots are designed to work beside humans in industrial factories using probabilistic algorithms. Examples therefor are the "Mobile Industrial Robots (MiR)" [1] and the "Kuka KMR iiwa" [2]. These mobile robots are mostly using a safety Light Detection and Ranging (LiDAR) system. The problem with this probabilistic method is that there are currently no standards for mobile robots in an industrial environment. However, the standards are now in work and the TÜV Austria accepts these probabilistic methods only, if the mobile robot has a safety ring around it, which will guarantee a safe obstacle detection. 
On the other side were also different mobile robots using a more robust navigation. Two examples are the "Amazon Kiva Robots" and the "Alibaba Quicktron Robots". These robots are implemented in a human free industrial factory. Therefore, they do not have sensors to prevent obstacles like humans. These robots are working within a factory were an array of QR-Codes is implemented. The robots drive over these QR-Codes and therefore the robot knows the exact pose of itself in the factory. A pose of these mobile robots is defined with their cartesian coordinates in $\mathrm{x}$ and $\mathrm{y}$ and an orientation around $\mathrm{z}$ [3] [4].

The development area for this work is the digital factory of the UAS Technikum Vienna. It is an area with approximately $40 \mathrm{~m}^{2}$ and a variety of industrial robot of different companies. Further, half of the walls in the digital factory are large glass fronts, which makes it challenging for mobile robotics. The mobile robot MiR100 [5] will be used for transportation and manipulation applications. Furthermore, it uses two SICK Laser scanners for SLAM and obstacle avoidance it does not identify all hazard situations, which will be further discussed in this paper.

In the following work, three different concepts will be shown, which should be certifiable of the TÜV Austria. Therefore, it is necessary to obey the rules of all standards, which has a link with mobile robots and functional safety criteria.

\section{Safety Ring for mobile robots}

To guarantee a safe mobile robot for the industry, which works in the same area as humans without many restrictions or fences, a safety ring for mobile robots is in development. The safety ring must guarantee to detect any obstacle in the maximum breaking distance of a mobile robot. If any obstacle is in danger distance of the safety ring, a safety controller applies an emergency stop and turns off the motors of the mobile robot.

Probabilistic is an essential subject in mobile robotics for their localisation and navigation [6]. Due to a safety ring a variety of new approaches for state-of-the-art probabilistic robotics could find permission to get used in industry environments. Thus, a wide spectrum for self-navigation of a mobile robot is possible. Furthermore, non-tactile sensors like LiDAR makes it possible for probabilistic approaches. In addition, the safety ring should be a modular system, which can be implemented to every mobile robot for the industry and can guarantee a safe working area for humans and robots.

The safety ring will be implemented on a state-of-the-art mobile robot at the UAS Technikum Vienna. More precisely on the MiR100. This mobile robot can be arbitrary upgraded on its top. In this case, for different research projects, the MiR100 got upgraded with an aluminium framework and a Universal Robot (UR) 5 on the top of it. But this additional framework makes the MiR100 not safe for every situation. The MiR100 has two SICK S300 Professional laser scanners, an Intel RealSense 3D camera and four Ultrasonic safety sensors to detect obstacles, but they are designed for a height of the robot of $995 \mathrm{~mm}$. With the UR5 mounted on the mobile robot, the height is more than $995 \mathrm{~mm}$, so additional safety sensors are needed to detect obstacles, which would crash into the aluminium framework or even into the UR5.

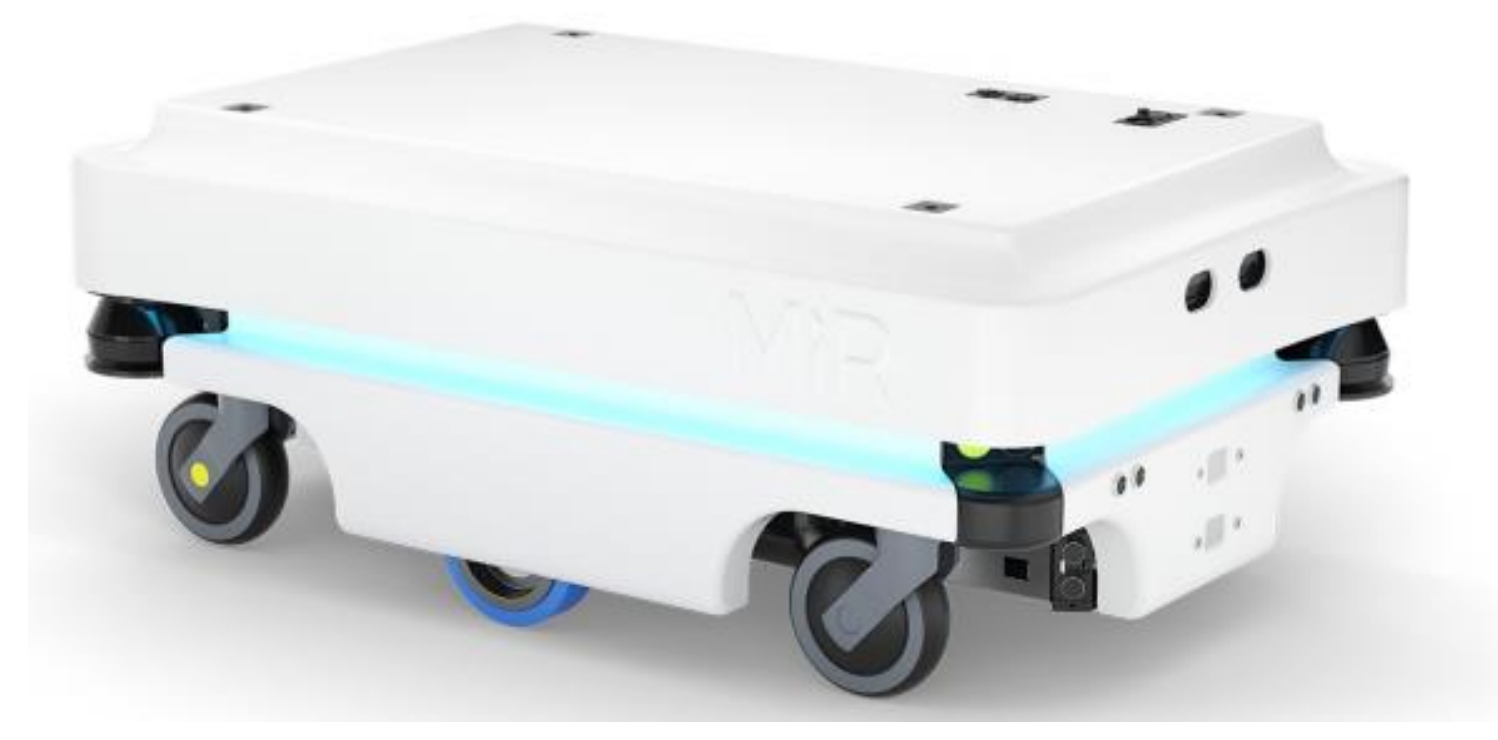

Fig. 1. Mobile robot MiR100 - Available in digital factory of UAS Technikum Vienna [5]

That is not the only motivation to implement a safety ring for this mobile robot. The safety of the mobile robot is also not guaranteed at fall down areas like stairs or steep ramps, so additional ground sensors to detect dangerous fall down areas are needed. Finally, some additional options can be implemented at the MiR100. If a human will not be detected there will be a barrier implemented to counter an any harm for human or robot. Furthermore, tactile sensors getting implemented to the robot. Therefore, around the mobile robot some safety bumpers will be the last opportunity to counter a hazard situation for humans. 


\subsection{Relevant standards}

To get a safety certificated system, it is important to get an overview about all standards and norms about the topic mobile robot in industrial environment and to obey them. Unfortunately, there is no specific standard (Type C-Norm) for this exact topic, which is why the general norm about machine safety (ISO 12100) becomes effective [7].

According ISO 12100 [8] all limits of the machine, in this case of the mobile robot, must be defined. Next, a hazard identification of the mobile robot must be revealed and afterwards a risk estimation or risk analysis must be done. After these three steps, a risk evaluation is following. When the risk evaluation is finalized, it must be reviewed if the risks are sufficient prevented. If it is not enough prevented, it has to be proven, if the risk has been adequately reduced. If not, it has to be checked if the risk can be reduced by inherently safe design measures. If the hazard is still not removed, it must be checked if the risk can be reduced by guards or protective devices. If the risk reduction is still negative, the limits of the machine can be maybe specified again, if not an information for use guideline must be defined for this risk.

In addition, related norms can be considered for a safe mobile robot with an integrated safety ring. Also, the MiR100 referred to these standards and indicate them in their EU declaration of Conformity [5]. Especially the EN 1525 [9] Safety of industrial trucks - Driverless trucks and their systems and the EN 1175 [10] - Safety of industrial trucks Electrical requirements standing in relation to mobile robotics. Furthermore, EN 60204 [11] is indicated which contains the safety of machinery and its general requirements of electrical equipment of machines and EN 13849 [12] for safety related parts of control systems.

In case of functional safety EN 61508 [13] is a relevant standard for integrating safety systems for the safety ring.

\subsection{Obstacle Avoidance}

An important sensing for mobile robots are the perception, so it knows its environment. Therefore, obstacle avoidance sensors are especially for human's safety relevant subjects. Therefore, two time-of-flight sensors getting mentioned with safety certificate for human safety. They can be differentiating between electromagnetic and sound wave sensors [14].

Very standardly used electromagnetic time-of-flight wave sensor are laser sensors. They are often used as a LiDAR system and are typically used for SLAM. They have two benefits for mobile robots. First, it can localize itself in its environment. Secondly, it is an efficient way for obstacle avoidance. Therefore, it is essential where to mount the LiDAR system on the mobile robot. As example, the MiR100 has two LiDAR sensors diagonally implemented on the robot. Pros are that it is using electronics, which can resolve the information in picoseconds, which makes this obstacle avoidance very fast. The downside is that this makes these sensors very expensive [14].

The other time-of-flight sensor are ultrasonic sensors. The basic principle is quite the same as a laser sensor, which receives the reflective wave of the sensor and calculates the time of traveling to the object and backwards. Especially for glass walls, it is useful to use ultrasonic sensors, because laser or infrared is not reflective for glass and can be detected. A disadvantage of ultrasonic sensors could be an annoying beat while calculating the distance to other objects [14].

\subsection{Floor detection sensors}

The same sensor system for obstacle avoidance can be used to detect the floor underneath the mobile robot. Hazard areas for mobile robots are stairs, ramps or even slopes without barriers. Usually the working area of mobile robots are not in this kind of areas. However, to fulfil a safe mobile robot, which can drive everywhere and use every algorithm of mobile robotics, it is necessary to covering also these dangerous areas. Therefore, it is possible to implement ultrasonic sensors at the ground plate of the mobile robot to detect a floor while the mobile robot is driving. The distance to the robot should not get bigger than it is usually while driving on a planar ground. At a slope a dangerous distance to the floor must be calculated with the angle of the slope [15]. Infrared would also be a possibility for floor detection as well as obstacle avoidance but unfortunately there were no safety certificated infrared sensors found on market with an integrated receiver and transmitter for a safe application.

\subsection{Tactile sensors}

Tactile sensors for mobile robots are only the last left opportunity to protect humans or other safety relevant objects. Therefore, bumpers are ideal for this application. An advantage to implement them is to give a safety area between bumper and mobile robot, so the mobile robot doesn't have any contact with the safety relevant object, only with the bumper itself. If those tactile sensors recognize resistance, the motors of the mobile robot will be shut down immediately [14] [16].

\subsection{Frame safety sensors}

On the top of the MiR there is a UR5 robotic arm mounted. To avoid critical hazards, it would be possible to implement a light curtain to stop the arm when a person reaches into the working area of the robot. The same concept is also possible with the safety-certificated cameras, which have the same principle [17] [18]. 
However, to mount and implement the safety sensors on the mobile robot it would be necessary to extend the aluminium framework around the robotic arm. Therefore, all areas around the robotic arm can be covered against reaching into the working area. Thus, the flexibility and working area of the robotic arm is getting restricted because of the frame.

\subsection{Recommended concept}

The MiR100, where the safety ring should be implemented, has already sensors, which should guarantee safety. However, it has two SICK laser sensors for the safety of people because these sensors are the only one, which are certificated with a performance level and guarantee a functional safety function on the mobile robot. Although these are the only external sensors which are connected to the SICK safety system and are responsible for the safety of people. To guarantee additional safety with the mounted aluminium framework and the UR5 on the mobile robot, further safety sensors will be implemented. Nevertheless, the two SICK laser sensors are the basic building block for a safety ring. Nevertheless, the safety ring should be a modular system, which can be easily implemented on every mobile robot with a few adaptions. The safety control system can be seen as black box but the construction design, the wiring and the supply voltages can be different at various mobile robot systems.

First, the height of the whole mobile robot must be covered with the safety sensors. Therefore, additional two SICK laser scanners on each side of the mobile robot can be implemented with the sensor area looking upside. They can cover the height of the mobile robot and ensure that the robot is not driving into any object. With the already implemented laser scanners the top of the robot would not be fully covered. Thus, objects besides the mobile robot and objects, which are wider than the mobile robot, can be detected. To ensure the object detection in front of the robot, which are thinner than the mobile robot, a fail-safe ultrasonic sensor can be implemented in front and the back of the robot to cover its height.

Secondly, it is recommended to implement ground sensors for stairs or steep ramps. Usually mobile robots should not drive in places with stairs or steep ramps, but to cover a completely safe mobile robot it is necessary to implement such sensors. The recommendation therefore are ultrasonic distance sensors. There are already fail-safe ultrasonic sensors on market, which can guarantee a functional safety. As last instance, if all safety sensors should fail, safety bumpers will be implemented to the mobile robot. These tactile sensors can give feedback if they crash into an object and force the motors because of the safety control system to stop immediately [19].

Before the mobile robot can start driving to fulfil his jobs, the implemented robotic arm should drive to a safe home position and get shut down with an emergency stop trigger. Thus, there is no addition risk because the UR5 is not able to move. However, with this restriction additional safety sensors for the frame will not be implemented because this considers the manipulation with the mobile robot, but this should not be covered with the safety ring in this project. The ring should only cover collisions and hazards while driving in industrial environments.

\section{Safe Navigation for mobile Robots}

Another possible safety concept for a mobile robot will be presented in this chapter. Maybe a safety ring cannot be implemented because of financial or space limitations. Furthermore, it could also happen that the hazard of the probabilistic robotic navigation stays too high for the "TüV Austria". A logical decision tree would be better, because a decision would deliver every time the same result with the same input parameters. Whereas it is not defined in a probabilistic function. Therefore, this chapter treats the safe navigation for mobile robots under use of logical decisions. The robust navigation of autonomous mobile systems is a big research area, which can be divided into the areas of selflocalization, map building and path planning [20].

However, it is important to differentiate between an autonomous mobile robot (AMR) and an automated guided vehicle (AGV) first. AMRs do not have the need of a defined track, because it navigates dynamically in the environment. A map can be built automatically (SLAM) or saved in the robot, which allows it to plan its own path in the factory. Further, it is easy to expand or change the work area, because only the map must be updated. In contrast to the AMR, an AGV requires "tracks", in form of lines or figures on the floor. Therefore, it is not as easy and time consuming, to expand or change the work area. Because of this, the AGV is restricted to fix routes and stops at any obstacle without the possibility to change the route [21].

Nevertheless, probabilistic methods cannot fully guarantee a safe navigation. The resolution of the SICK S300 Professional used on the MIR100 have a configurable resolution of 30-150mm [22]. However, another AMR producer named "Neobotix" indicates a localization accuracy of approximately 200mm [23]. For safe navigation it is not allowed, that the AMR diverge this much from the real position. Therefore, this concept combines the old but proven methods of the AGV's with the modern approaches of the AMR's.

For the self-localization, many different sensor methods are available. Further, not each sensor can be used. It must be guaranteed, that the sensor output is valid. Therefore, safety sensors which can deliver results reliable are very important. For the safe navigation, the sensors do not have to be always the extremely expensive safety sensors for personal protection. An overview about some of these sensors and the possible navigation methods will be given in this chapter. Sensors can be differentiated into extern and intern sensors. Intern sensors measure all data from the inside of the robot like the odometry, position, orientation or velocity. On the other side extern sensors receive data from the outside of the robot. For the optimal safe mobile robot concept, intern sensors as well as extern sensors must be used [16]. 
In Fig. 2 is the layout of the digital factory of the UAS Technikum Vienna shown. A few industrial robots should be reached by the mobile robot. Additionally, the robot should follow in this concept only defined paths drawn in the figure. These paths should also be taped on the floor and on critical places there must also be traffic lights to alert the employees of an approaching mobile robot. In the first concept and because of the lack of space only one MIR100 should drive in the factory.

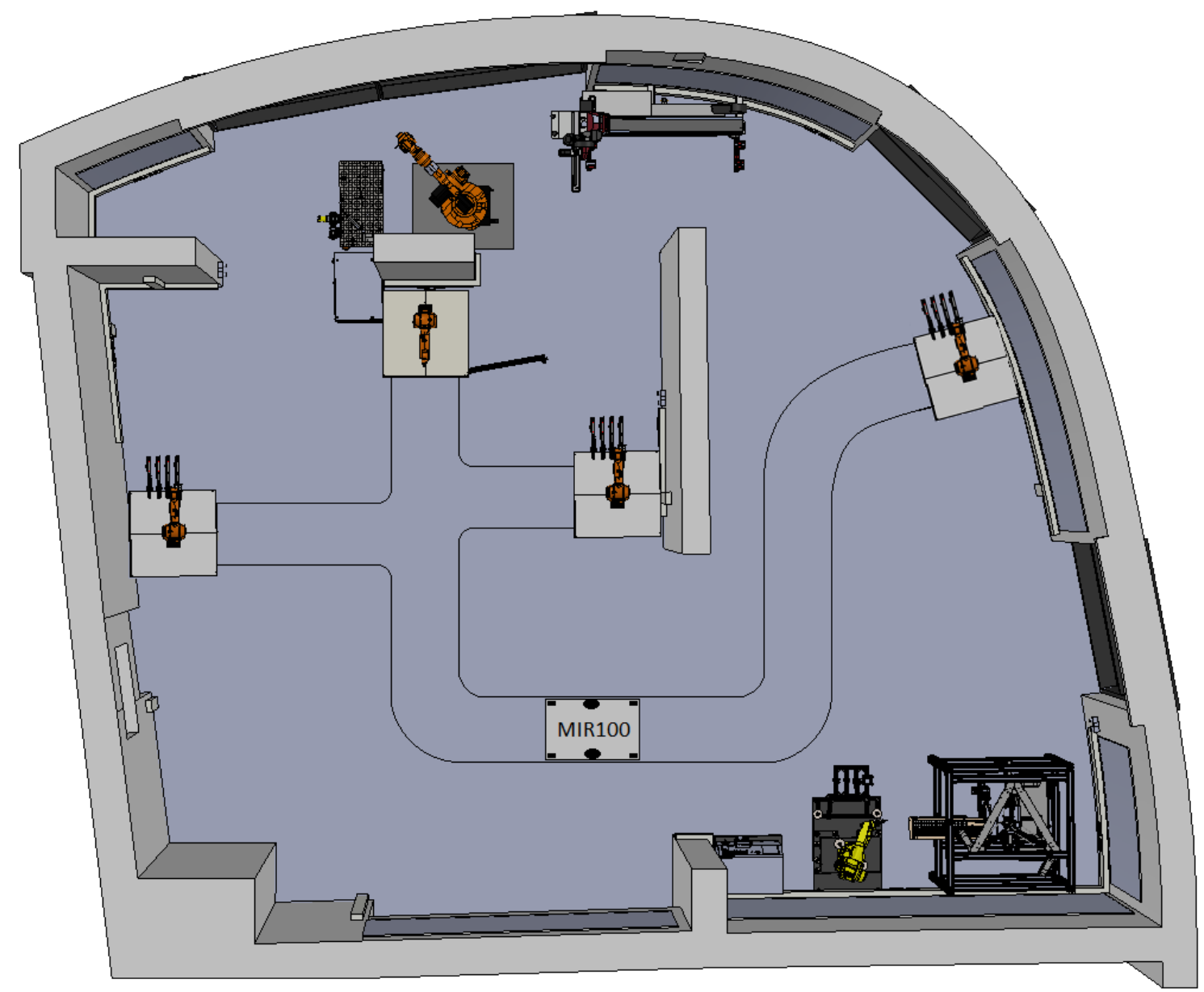

Fig. 2. Concept and layout of the digital factory of the UAS Technikum Vienna

\subsection{Intern Sensors}

Some internal sensors would be very interesting in combination with the following presented external sensors. For example, could an encoder be used for the odometry to measure the distance from one point to another point in the map. The odometer alone will not be very accurate, because the actual position will drift without global information of their position. But it could be used for control of the actual position with multiple sensors. Other potential interesting sensors could be compasses, gyroscopes or inertial measurement units (IMU). Many of these intern sensors are already implemented in available mobile robots [6] [16] [24].

\subsection{Guidepath following}

Guidepath following is a method most AGVs are using. The method is very simple and cheap, because the robot follows a predefined path strictly. In the simplest form the path could be an adhesive tape on the floor. Optical line sensors work with light to detect the adhesive tape and therefore the large glass fronts could be problematic. Magnetic line sensors could work around this problem with magnetic tape on the floor. With this method, a large amount of AMR could work trouble-free in the same factory, because the AMR could not get lost and a central PC knows exactly the position of the robots at any time. The biggest problem with this method is the lack of flexibility. Each new path must be taped on the floor and the digital representation must be updated on the system. However, this disadvantage for an AMR could be a perfect solution for the safe navigation, because the flexibility of the AMR path planning and obstacle avoidance is not wished. The workers should know the exact movement of the robot and should not be surprised of flexible reactions and free path planning [24]. 


\subsection{Grid localization}

As mentioned in the introduction, the "Amazon Kiva Robots" and the "Alibaba Quicktron Robots" are using this navigation method. A grid of QR-Codes will be taped on the floor or the ceiling, whereby the distance between different QR-Codes should be very small. The mobile robots are scanning these QR-Codes, in which the label and position in the factory is stored. Industrial cameras are used for reliable scans and will extract the drift of the robot as position and orientation. The robot can now correct the drift and drive through the next QR-Code with the distance of a safety encoder. Alternatively, RFID- or NFC-Tags could be used instead of the QR-Codes. But only with the QR-Codes the drift orientation can be measured [3] [4] [25].

\subsection{Landmarks}

Landmarks work likewise as the grid localization but with a lower amount of needed marks. Landmarks must be placed well visible in the factory. One possibility would be the detection of the QR-Codes with an industrial camera. Whereby, the QR-Codes will be taped on walls or in view area in front of the robot instead of the ceiling or floor. Another possible landmark detection would be over a LiDAR sensor. The available MIR100 uses this already in form of V-and VL-Marker to detect positions like the docking station in the factory [26] [27]. However, it is also possible to use natural landmarks in an unmodified environment with an industrial camera for the localization. Different significant signs or planar landmarks as walls, doors and ceilings could be used for this kind of landmark localization [28] [29] [41].

\subsection{Triangulation}

Triangulation in mobile robotic is a geometric algorithm. At least three different well-defined emitters must be in range. Either the emitter sends a signal, or the emitter are working as reflector or landmark. With the distance to each of at least three emitters it is possible to calculate the current position of the mobile robot in a global map. One of the cheapest opportunities would be the usage of WLAN-routers as emitters. There exist already many routers in factories or indoor environment, which positions are known and will not be changed often [30]. Another possibility would be the installation of wireless sensor networks. Most of them are working with ultra-wideband (UWB) technology, which is optimal in indoor environments because of their wavelength [31] [32].

The producer "posyx" has invited an easy to implement indoor GPS technology based on ultra-wideband emitting nodes. In the specifications, a positioning accuracy of a few centimetres will be guaranteed [33]. However, to use the triangulation method a Kalman filter must be used, because of the position noise. Otherwise, the mobile robot will never position correctly and tremble on the correct position [34].

\subsection{Saved distance map}

The probabilistic method of particle filter could not be used for this concept, because of its uncertainty [6]. However, as alternative method a saved distance map could be used. Therefore, a map of the factory must be stored in the mobile robot. The uniqueness of this map would be, that the position of the mobile robot to the walls and obstacles in each position of the factory will be measured. Afterward it would be possible to lookup the continuous measurements of the robot in the saved distance map and detect the position. Maybe different positions of the factory have similar saved distances. Therefore, virtual agents would be necessary. Each possible position of the mobile robot would be represented from one virtual agent. After the mobile robot is moving more and more virtual agents would get lost, because the position could not be there based on the new measurements. Time-of-Flight sensors as LiDAR, Ultrasonic- or Infrared distance sensors would be used [35].

\subsection{Smart floor}

The last method presented for the concept of localization would be a smart floor. This smart floor would be based on pressure-sensitive plates like the "PSENmat" from "PILZ". Either the whole factory, only the path of the mobile robot or the hazardous intersection could be armed with these plates. The system would know exactly on which plate the mobile robot is driving currently. If one plate will be pressed without the movement of a mobile robot, it could be a human and the mobile robot or the system could react appropriate [36].

\subsection{Digital representation of the factory and navigation algorithm}

Many possible localization methods were described. However, the navigation can be divided into self-localization, map building and path planning [20]. Therefore, this section will now treat the map and the path planning. The map could be represented digital as 2D-matrix or tree form. The position and possible ways of the robot would be clearer in the matrix. However, it is not easy to expand the map, because the matrix must be expanded in each direction and many problems within the path-searching algorithm can be occur afterwards. 
An easier solution for the extension would be the representation over the tree form, because a new station or way could easy be added on an existing branch of the tree. Each SLAM algorithm and self-creating or dynamic map would use probabilistic and is therefore also not allowed in this concept. If the map exists in digital form on the robot, an optimal path-searching algorithm could be selected. However, it is unnecessary to mention, that probabilistic methods are not allowed again. But there are logical path-planning algorithms as depth-first, breadth-first or A*. Each of these algorithms will find a solution if there is one. Nevertheless, the time consumed finding a path will be different [37] [6].

\subsection{Recommended Concept}

A MIR100 and a "posyx" Indoor-GPS-System is already available in the factory. Additionally, to the SICK LiDAR sensors and the Indoor-GPS-System, a magnetic line sensor will be used to locate the robot within $5 \mathrm{~cm}$. The line-following is one of the best methods, because the robot will stop without seeing a line automatically. Further, there are many glass fronts in the factory and therefore the magnetic line sensor will be more reliable than the optical line sensor. The map would be represented as a tree structure, because it is easier to expand the map. And the A*-Algorithm would be used for the path planning, because it will find the fastest way to the destination, if there is one. With predefined magnetic lines, this combination will be the most reliable possibility.

\section{Aware Environment through sensors}

There is a trade-off in the two methods mentioned before. Three categories are used to design a robot usually. It can be safe, performant and low-cost. Since a mobile robot should always be safe, this category can't be disregarded. Therefore, two different type of mobile robots are possible for the concepts presented in the chapters above. On the one hand, cheap sensors could be used, but the robot lose its performance since it must drive slower. On the other hand, expensive sensors with a wider and safer scan area could be used at higher performance expressed in higher working speed. [37] treats the possibility to create a mobile robot using all three categories at its maximum possible value. For this, he made an aware environment out of the factory. Environment build-in sensors recognise human and tracking them. This method is called a virtual sensor for the robot, because he can use the sensor data of the aware environment for a safe navigation. The sensor methods can be differentiated into passive and active. Because employees do not wear active tags permanently on body and the system would then not be safe enough a passive system without the need of wearing any tag will be better and safer. In the cited reference a passive infrared wireless sensor node was used for this problem.

Wireless sensor networks (WSN) are one common way in today's research area for this kind of virtual sensors. GPS is not working in an indoor environment and therefore WSNs are getting used. Section 3.5 treated the possibility of the WSNs for an indoor localization. However, with suiting sensors equipped WSNs could also detect humans and communicate with the system wirelessly. Many factories already have WSN in usage or if they would equip the factory later, the WSN could be used for localization and human tracking in both ways, which is a big advantage. Another advantage would be the lower investments at a high amount of applied mobile robots. This happens through the fact, that the sensors normally build in each mobile robot are outsourced in the WSN. Therefore, a single investment in the WSNs could save many investments in each mobile robot [37] [31] [32].

In Fig. 3 the overview of the wireless sensor network concept is shown. Multiple sensor nodes are connected over a wireless sensor network, whereby each node contains sensors and information about their position in the factory and other useful informations. Each target node information can be accessed by a sink node in the wireless sensor network. These wireless sensor nodes could be WLAN-routers or simple and cheap microcontroller with additionally sensors. [37]

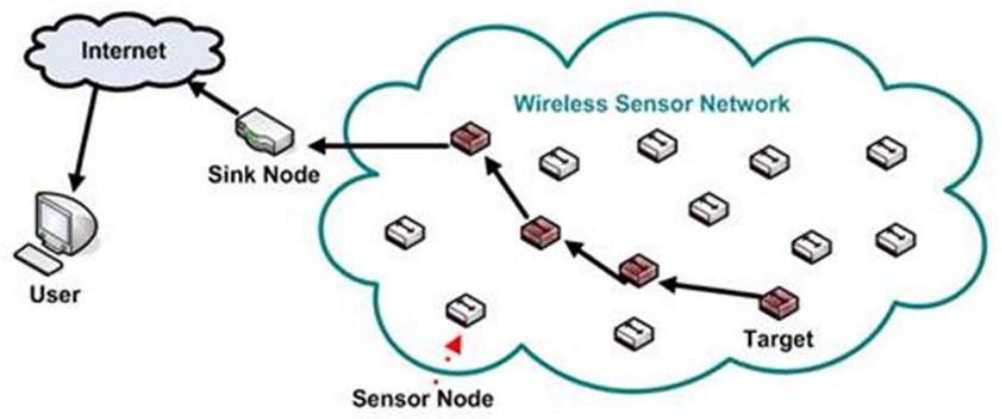

Fig. 3. Overview of the wireless sensor network concept [40]

Another possibility could be a smart floor. As already mentioned in section 3.7 a pressure-sensitive floor could exactly locate the humans, obstacles and mobile robots in a factory. However, at the time of this paper a suited sensor solution was not found. Pressure-sensitive plates like the "PSENmat" from "PILZ" are mostly used only in front of industrial robots and it is possible to connect up to 64 plates. Nevertheless, the available sensors are not built for a complete factory floor [36]. 
Of course, it would be possible to track the people from above instead of a smart floor. Therefore, a RGB-D camera could be mounted on the ceiling and detect and track human motion [38]. However, a camera may harm the privacy because the employees or other humans could be tracked against their willing [37]. Especially today, the respect of privacy is more important than ever. Considering that, the tracking with a passive or pyroelectric infrared (PIR) sensor would be better [39].

\section{Results and Discussion}

In general, it can be said that the safety ring is the best solution to implement, because it guarantees a safe environment for safety relevant objects and mobile robots. With this system the mobile robot shouldn't harm any objects because of the safety certificated and partially redundant used sensors. Also, the safety ring will be a modular concept which makes it possible to adapt for every mobile robot in industry. Furthermore, state of the art approaches of probabilistic robotics can always be tested and used with the safety ring and the safety control system should prevent injuries for any safety relevant objects.

The concept of a safe navigation is still not far proceeded and obeys to the line following. This makes not much difference to an $\mathrm{AGV}$ and restricts a lot of new approaches of mobile robotics. However, it is one of the most reliable methods. Because of the logical decision, if the mobile robot is on the line or not, the state is clear at any time. Furthermore, the start position is defined on one position, without the problem of multiple virtual agents or particles.

As written before, the most modern navigation methods are using probabilistic methods. With safer sensors and more reliable software methods, it could be possible, that also modern approaches could be used in the future. The implantation cost of this concept would be cheap, because most of the mobile robots already have line following sensors, LiDAR systems or distance sensors.

The concept of the aware environment is currently not safe enough to get a certificated environment. This is because of the probabilistic and not logical approach to track people in this environment. Moreover, the investment costs for this concept in an industrial area can be more expensive than to implement a safety ring or a safe navigation to a mobile robot. Further, this concept has the big advantage that the costs only occur once. Moreover, in a large environment with many mobile robots it could be the cheapest method. The implementation costs could also be cheaper, if the environment already uses some sensors to track persons.

Finally, the selection criteria for a safety concept always implies the application for the mobile robot, the amount of applied mobile robots and the layout of the industrial environment. The acquisition costs of an aware environment can be very high. Nevertheless, in comparison to the costs of at least twenty mobile robots with a safety ring or safe navigation, it can be the cheapest solution. The next table is a comparison and estimation about the usage of all three concepts for a safe industrial environment using mobile robots:

\begin{tabular}{|c|c|c|c|}
\hline Criteria & Safety ring & Safe navigation & Aware environment \\
\hline Overall safety & + & o & - \\
\hline Possibility for state-of-the-art approaches & + & - & o \\
\hline Implementation costs & - & + & $\mathrm{O} /-$ \\
\hline Maintenance costs & $\mathrm{O}$ & $\mathrm{O}$ & o \\
\hline $\begin{array}{l}\text { Usefulness to implement in an industrial environment } \\
\text { with }>20 \text { mobile robots }\end{array}$ & - & o & + \\
\hline
\end{tabular}

Table 1. Comparison of all three concepts

\section{Conclusion and Future}

In this paper, three different safety concepts were made to design a safe environment in industrial facilities with mobile robots. The first concept includes the safety ring, which is responsible for the reliable object detection for different situations. The next concept treats the field of safe navigation. The focus was about robust self-localisation. Additionally, the fields of digital representation of the factory and path planning were discussed. The last concept covers the topic of aware environment succinct. The next steps are to implement the concepts of a safety ring and a safe navigation on the MiR100. These two concepts will be evaluated against each other after the implementation to prove the advantages and disadvantages of chapter 5. Perhaps, also the last concept of the aware environment will be realized afterwards.

\section{Acknowledgments}

We would like to thank the "MA23" and the UAS Technikum Wien for the opportunity to work on this paper and its very interesting topic. This accomplishment would not have been possible without them and we appreciate it. 


\section{References}

[1] MiR, (2017). The future in motion. Available from: https://www.mobile-industrial-robots.com/de/ Accessed: 201809-10

[2] KUKA, (2018). KMR iiwa. Available from: https://www.kuka.com/de-at/produkte-leistungen/mobility/mobileroboter/kmr-iiwa Accessed: 2018-09-10

[3] Dailymail. (2017). Wifi-equipped robots triple work efficiency at the warehouse of the world's largest online retailer, Available from: http:/www.dailymail.co.uk/news/article-4754078/China-s-largest-smart-warehouse-manned-60robots.html Accessed: 2018-09-07

[4] Memmesheimer, R. (2015). Lagerhaus-Automatisierung am Beispiel Kiva Systems, Available from: https://www.uni-koblenz-landau.de/de/koblenz/fb4/ist/AGZoebel/Lehre/Sommer2015/SeminarASidA/TA7 Accessed: 2018-09-05

[5] MIR (2018). MiR100 User Guide, Available from: http://www.mobile-industrial-robots.com/media/4048/mir100user-guide_robot-interface-20-v11.pdf Accessed: 2018-09-09

[6] Thrun, S.; Burgard, W. \& Fox, D. (2005). Probabilistic Robotics, The MIT Press, ISBN: 0262201623, London, England

[7] Leuze eletronic, (2018). Hierachy of European standards of machine safety. Available from: https://leuze.de/en/deutschland/loesungen/anwenderwissen/arbeitssicherheit/2_maschinensicherheit_in_der_eu/2_ 2_3_hierarchie_der_europaeischen_normen_zur_maschinensicherheit/2_2_3_hierarchie_der_europaeischen_norm en_zur_maschinensicherheit.php?lang=eng Accessed: 2018-09-09

[8] DIN EN ISO 12100:2011, (2011). Safety of machinery - General principles for design - Risk assessment and risk reduction

[9] DIN EN 1525:1997, (1997). Safety of industrial trucks - Driverless truicks and their systems

[10] DIN EN 1175:2011, (2011). Sicherheit von Flurförderzeugen - Elektrische Anforderungen - Teil 1: Allgemeine Anforderungen für Flurförderzeuge mit batterieelektrischem Antrieb

[11] DIN EN 60204:2014, (2014). Sicherheit von Maschinen - Elektrische Ausrüstung von Maschinen

[12] DIN EN ISO 13849:2016, (2016). Sicherheit von Maschinen - Sicherheitsbezogene Teile von Steuerungen

[13] DIN EN 61508:2011, (2011). Funktionale Sicherheit sicherheitsbezogener elektrischer/elektronischer/programmierbarer elektronischer Systeme

[14] R. Siegwart; I. Nourbakhsh, (2004). Introduction to Autonomous Mobile Robot, The MIT Press, ISBN: 9780262195027, London, England

[15] B. Wei, J. Gao, K. Li, und H. Chen. (2009). Navigation and slope detection system design for autonomous mobile robot, Available from: https://ieeexplore.ieee.org/document/5274672/ Accessed: 2018-09-09

[16] Pieper, S. (2007). Sensoren und Aktoren von autonomen Robotern, Ph.D. Dissertation, Institut für Technik und ihre Didaktik, Westfälische Wilhelms Universität Münster, Germany

[17] SICK. (2018). Sicherheits-Lichtvorhänge, Available from: https://www.sick.com/at/de/optoelektronischeschutzeinrichtungen/sicherheits-lichtvorhaenge/c/g185751 Accessed: 2018-09-09

[18] SICK. (2018). Sichere Kamerasysteme, Available from: https://www.sick.com/at/de/optoelektronischeschutzeinrichtungen/sichere-kamerasysteme/c/g192052 Accessed: 2018-09-09

[19] Ullrich, G. (2011). Fahrerlose Transportsysteme: eine Fibel - mit Praxisanwendungen - zur Technik - für die Planung, Vieweg + Teubner, ISBN 978-3-8348-0791-5, Berlin, Germany.

[20] Gutmann, J.S. (1999). Robuste Navigation autonomer mobiler Systeme, Ph.D. Dissertation, Fakultät für Angewandte Wissenschaften, Universität Freiburg, Germany

[21] MIR. (2017). AGV vs. AMR - What's the Difference, Available from: https://www.mobile-industrialrobots.com/en/resources/whitepapers/agv-vs-amr-whats-the-difference/ Accessed: 2018-09-04

[22] SICK. (2018). S300 Professional Datasheet, Available https://www.sick.com/media/pdf/9/49/849/dataSheet_S30B-3011DA_1056429_de.pdf Accessed: 2018-09-07

[23] Neobotix. (2018). Mögliche Lokalisierungsmethoden für Neobotix PlatformCtrl, Avaiable from: https://www.neobotix-roboter.de/fileadmin/files/downloads/Allgemeines/Lokalisierungsmethoden.pdf Accessed: 2018-09-07

[24] Everett, H.R. (1995). "Sensors for mobile robots", A.K. Peters, Ltd., ISBN: 1-56881-048-2, Wellesley, MA, USA

[25] Zhang, H.; Zhang, C.; Yang, W. \& Chen, C. (2015). Localization and navigation using QR code for mobile robot in indoor environment. Proceeding of IEEE International Conference on Robotics and Biomimetics (ROBIO) December 6-9, 2015, pp. 2501-2506, Zhuhai, China, DOI: 10.1109/ROBIO.2015.7419715

[26] MiR. (2016). Docking station - Technical Documentation, Available from: https://www.mobile-industrialrobots.com/media/1344/mircharge-user-guide-12.pdf Accessed: 2018-09-05

[27] Narayana, K.; Goulette, F. \& Steux, B. (2010). Planar landmark detection using a specific arrangement of LIDAR scanners, Proceedings of IEEE/ION Position, Location and Navigation Symposium, Indian Wells, CA, pp. 10571069, DOI: 10.1109/PLANS.2010.5507336 
[28] Se, S.; Lowe, D. \& Little, J.J. (2001). Vision-based mobile robot localization and mapping using scale-invariant features. Proceedings of IEEE International Conference on Robotics and Automation (ICRA) Vol. 2, pp. 2051 2058, DOI: 10.1109/ROBOT.2001.932909

[29] Li, Q.; Zhu, J.; Liu, T.; Garibaldi, J.; Li, Q. \& Qiu, G. (2017). Visual landmark sequence-based indoor localization, Proceedings of GeoAl'17:1st ACM SIGSPATIAL Workshop on Artificial Intelligence and Deep Learning for Geographic Knowledge Discovery (GeoAl'17) November 7-10, Los Angeles Area, CA, USA, pp. 14-23. DOI: $10.1145 / 3149808.3149812$.

[30] Röhrig, C. \& Künemund, F. (2008). WLAN based Pose Estimation for Mobile Robots, Proceedings of International Federation of Automatic Control (IFAC) Volume 41, July 6-11, Seoul, Korea, pp. 10433-10438, DOI: 10.3182/20080706-5-KR-1001.01768

[31] Simon, J. \& Martinovic, G. (2013). Navigation of Mobile Robots Using WSN's RSSI Parameter and Potential Field Method. Acta Polytechnica Hungarica Vol. 10, November 4, pp. 107-118.

[32] Sun, X.; Zhao, X.; Li, E.; Li, H. \& Liang Z. (2010), Mobile Robot Navigation Using RSSI Potential Field in Wireless Sensor Network, Journal of Computational Information Systems 6:14, pp. 4751-4759

[33] Posyx. (2018), Accurate positioning - Ultra-wideband technology, Available from: https://www.pozyx.io/ Accessed: 2018-09-05

[34] Font, J.M. \& Batlle, J.A. (2012). Dynamic triangulation for mobile robot localization using an angular state Kalman filter., Available from: http://www.cim.mcgill.ca/ font/downloads/ECMR05_1.pdf Accessed: 2018-09-05

[35] Russel, S.J. \& Norvig, P. (2016), Artificial intelligence: a modern approach, Third Edition, Pearson, ISBN: 9781292153964, Upper Saddle River, New Jersey

[36] Pilz. (2018). Sicherheitsschaltmatte PSENmat, Available from: https://www.pilz.com/deAT/eshop/00106002247124/PSENmat-Sicherheitsschaltmatte Accessed: 2018-09-05

[37] Arndt, M. (2016). Safe and cost-efficient mobile robot navigation in aware environments, Ph.D. Dissertation, Fachbereich Informatik, Technischen Universität, Kaiserslautern, Germany

[38] Munaro M.; Lewis C.; Chambers D.; Hvass P. \& Menegatti E. (2016) RGB-D Human Detection and Tracking for Industrial Environments. Proceedings of the 13th International Conference Intelligent Autonomous Systems (IAS) 13, pp 1655-1668, DOI: 10.1007/978-3-319-08338-4_119

[39] Yun, J., \& Lee, S.-S. (2014). Human Movement Detection and Identification Using Pyroelectric Infrared Sensors. Sensors. Sensors, Vol. 14, No. 5, May 2014, pp. 8057-8081. DOI: 10.3390/s140508057

[40] Puyalnithi, T. \& Viswanatham. M. (2016). Visualization and Statistical Analysis of Multi Dimensional Data of Wireless Sensor Networks Using Self Organising Maps. International Journal of Engineering and Technology 8, pp. 391-395.

[41] Crnokić. B.; Rezić. S. \& Pehar. S. (2016). Comparision of Edge Detection Methods for Obstacles Detection in a Mobile Robot Environment, Proceedings of the 27th DAAAM International Symposium, pp.0235-0244, B. Katalinic (Ed.), Published by DAAAM International, ISBN 978-3-902734-08-2, ISSN 1726-9679, Vienna, Austria, DOI: $10.2507 / 27$ th.daaam.proceedings.035 